Low-pressure gas breakdown in longitudinal combined electric fields

This article has been downloaded from IOPscience. Please scroll down to see the full text article.

2010 J. Phys. D: Appl. Phys. 43425202

(http://iopscience.iop.org/0022-3727/43/42/425202)

View the table of contents for this issue, or go to the journal homepage for more

Download details:

IP Address: 109.87.54.56

The article was downloaded on 10/10/2010 at 16:51

Please note that terms and conditions apply. 


\title{
Low-pressure gas breakdown in longitudinal combined electric fields
}

\author{
V A Lisovskiy, N D Kharchenko and V D Yegorenkov
}

Kharkov National University, 4 Svobody sq., Kharkov 61077, Ukraine

E-mail: lisovskiy@yahoo.com

Received 7 February 2010, in final form 7 September 2010

Published 5 October 2010

Online at stacks.iop.org/JPhysD/43/425202

\begin{abstract}
This paper contains the complete experimental and analytical picture of gas breakdown in combined electric fields for arbitrary values of $\mathrm{rf}$ and dc fields. To obtain it, we continued the study of the discharge ignition modes in nitrogen with simultaneous application of $\mathrm{dc}$ and $\mathrm{rf}$ electric fields presented in Lisovskiy et al (2008 J. Phys. D: Appl. Phys. 41 125207). To this end, we studied the effect of rf voltage on dc discharge ignition. When we applied an rf voltage exceeding the one corresponding to the minimum breakdown voltage of a self-sustained rf discharge, the curve of dependence of the dc breakdown voltage of a combined discharge on gas pressure was found to consist of two sections. We got the generalized gas breakdown criterion in the combined field valid for arbitrary values of rf and dc electric fields. The calculation results agree with experimental data satisfactorily.
\end{abstract}

\section{Introduction}

A gas discharge in combined fields when radio frequency (rf) and direct current $(\mathrm{dc})$ voltages are applied simultaneously to the same electrodes is employed in various plasma technologies, e.g. for etching different materials, plasma cleaning of technological discharge chambers, in plasma chemistry, for pumping gas lasers, for sterilizing medical tools, etc [1-5]. For example, Lai et al [1] demonstrated that applying an auxiliary negative potential to the rf electrode increases the discharge plasma concentration and accelerates processes of etching semiconductor plates and modifying surfaces of materials. Dc voltage application to a burning rf capacitive discharge permitted us to introduce up to $27 \mathrm{MW}$ power into a $\mathrm{CO}_{2}$ gas discharge plasma [3,4]. A combination of $\mathrm{dc}$ and $\mathrm{rf}$ electric fields improves the stability of gas discharge burning and permits the deposition of high power into the discharge which is important for achieving optimum conditions of performing technological processes [3-7]. In order to optimize plasma technology we should also know the conditions for igniting a combined discharge in various gases and with different combinations of dc and rf voltages applied across a discharge gap. Available papers contain a broad range of conditions for igniting a self-sustained $\mathrm{rf}$ discharge in various gases (see, e.g., [8-11] and the references cited therein). Dc discharge ignition is also studied in sufficient detail, e.g., in [12-16]. But the question of igniting a longitudinal combined discharge was not subjected to such a broad study. Nevertheless, the author of [17] noticed an increase in $\mathrm{rf}$ breakdown voltage when the dc voltage was simultaneously applied across the discharge gap. The author of [18] remarked that he had discovered an increase in the breakdown rf voltage in the combined electric fields even earlier [19]. Moreover, it was also shown in experiment [19] that the rf discharge can be extinguished by the application of a dc voltage across it. Brown [20] has demonstrated in theory that the dc voltage application increases the microwave breakdown field. The authors of [21] studied the ignition of a transverse combined discharge in crossed rf and dc electric fields. Reference [10] demonstrated that application of a dc voltage across the rf discharge with a frequency $f=20 \mathrm{MHz}$ involved an increase in the breakdown voltage for the $\mathrm{O}_{2}+\mathrm{CCl}_{4}$ mixture. Reference [11] also reported an increase in the rf breakdown voltage of the combined discharge compared with the self-sustained rf discharge. References [22,23] studied in experiment as well as with a numerical modelling the ignition of rf discharge combined with the dc electric field in the argon pressure range $p=1-10$ Torr for clean aluminium electrodes as well as for those covered with glass. They obtained that for clean aluminium electrodes the application of a dc voltage first increased the value of the breakdown rf voltage, but, starting from a certain critical value of the dc voltage, the rf breakdown 
voltage of the combined discharge decreased. This effect was not observed with the electrodes covered with glass. Reference [9] studied in experiment and theory the rf and longitudinal combined ( $\mathrm{rf}+\mathrm{dc}$ electric fields) breakdown in different gas species and for various inter-electrode gap values. It was suggested in this paper [9] how to distinguish the multipactor, Paschen and diffusion-drift branches in the rf discharge breakdown curve. A gas breakdown criterion in combined fields was also derived there taking into account the electron diffusion anisotropy. Reference [24] reported the results of gas breakdown modelling in combined fields taking into account the secondary electron emission from the electrodes. It also demonstrated the coincidence between the results of modelling and experiments presented in [25]. Reference [25] dealt with the discharge ignition in the longitudinal combined fields $(\mathrm{rf}+\mathrm{dc})$ both in experiment and theory. It presented the analytical model describing the gas breakdown in combined fields as well as the registered data for air in the discharge chamber with the inter-electrode gap values of $L=7.5$ and $23 \mathrm{~mm}$ and for argon with $L=23 \mathrm{~mm}$. The application of a small dc voltage together with the rf one increased the rf voltage of discharge ignition. However, [25] dealt only with the effect of the dc voltage on rf breakdown, whereas the effect of rf voltage on dc discharge ignition was not studied at all. Moreover, [25] does not contain the measured data on breakdown in nitrogen as well as in argon for narrow gaps. Reference [9] supplied the analytical gas breakdown criterion in longitudinal combined $\mathrm{rf}+\mathrm{dc}$ electric fields, taking into account the anisotropy of electron diffusion along and across the electric field direction. However, this criterion could predict the breakdown curves of the combined discharge only for a weak dc electric field not contributing to gas molecule ionization by electrons.

Apart from that, it is expedient to establish the combined discharge ignition parameters for small gaps $(L \sim 10 \mathrm{~mm})$ between electrodes because then the rf breakdown curves may possess not only the diffusion-drift branch but also the Paschen one. With large gaps an rf breakdown curve usually consists of only a diffusion-drift branch. Again, available references do not contain the results of studying the effect of rf voltage on gas breakdown in a dc electric field.

Therefore, this paper is devoted to studying the effect of rf voltage on dc discharge ignition as well as the effect of $\mathrm{dc}$ voltage on $\mathrm{rf}$ discharge ignition in nitrogen and argon. We demonstrate that if we apply for combined discharge ignition an rf voltage exceeding the minimum voltage for igniting a selfsustained rf discharge, then the dependence of dc breakdown voltage against gas pressure in a chamber consists of two sections. Similar to [26] we propose to distinguish three modes of combined discharge ignition depending on the relation between $\mathrm{dc}$ and $\mathrm{rf}$ voltages applied across the discharge gap: (1) ignition of an rf discharge perturbed by a dc electric field; (2) ignition in combined fields; (3) ignition of a dc discharge perturbed by an rf electric field. On the ground of the analytical model we got the gas breakdown criterion in combined fields being valid for arbitrary rf and dc voltage values and for predicting the registered data satisfactorily.

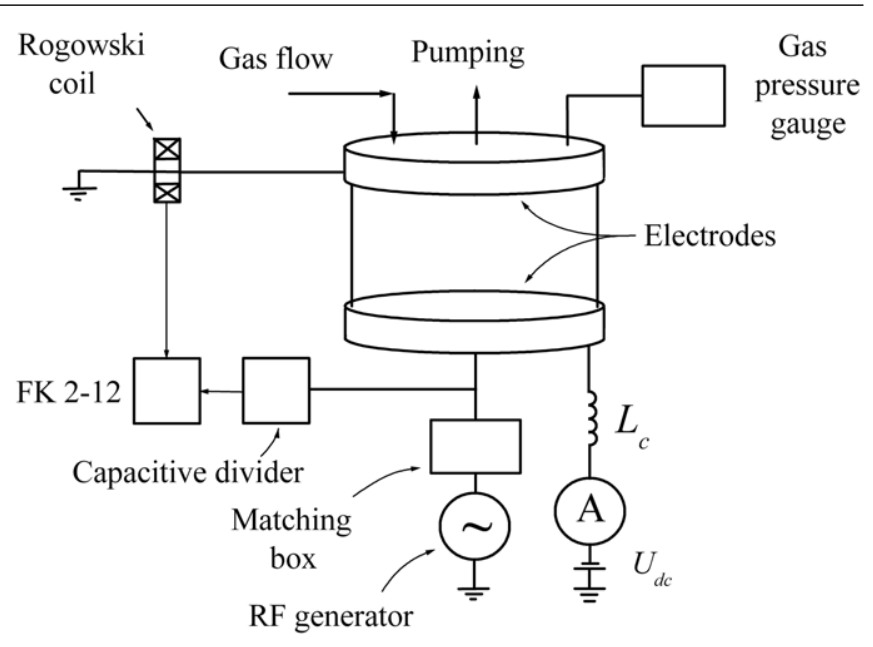

Figure 1. Scheme of the experimental setup.

\section{Experimental conditions}

Experiments were performed at nitrogen and argon pressures within the range $p=0.07-12$ Torr with amplitude values of rf voltage $U_{\text {rf }} \leqslant 2000 \mathrm{~V}$, dc voltage values $U_{\mathrm{dc}} \leqslant 1000 \mathrm{~V}$ and rf field frequency of $f=13.56 \mathrm{MHz}$. Figure 1 depicts our experimental setup schematically. Electrodes filled the cross section of a discharge tube of fused silica with an inner diameter of $100 \mathrm{~mm}$ which was vacuum-sealed between them. The gap between flat, plane parallel stainless-steel electrodes in the experiments with argon was $L=12$ and $32 \mathrm{~mm}$, whereas with nitrogen it was $L=10 \mathrm{~mm}$. We applied the rf potential to one of the electrodes whereas the other one was grounded. The rf electrode served as a 'cathode' simultaneously because a negative dc potential was applied to it.

A gas supply assembly fed the gas (of high $99.99 \%$ purity) through a set of small orifices in the grounded electrode. The discharge chamber was pumped out through a set of orifices in the same electrode. It provided a mode of feeding and pumping a gas uniformly over the electrode area, which plays an important role in technological processes. We employed a thermoelectric probe to register gas pressures in the range from $10^{-3}$ Torr to atmospheric pressure. Gas was pumped out with preliminary vacuum and turbo-molecular pumps giving a limiting vacuum order of $10^{-6}$ Torr. The rf generator was connected to the potential electrode through a matching box of П-type. A dc source was connected to the same electrode via a choke of $L_{\mathrm{c}}=4 \mathrm{mH}$ to avoid damage with rf current.

By 'breakdown voltage' we mean the maximum rf (or dc) voltage that can be applied across the electrodes without discharge ignition. The addition of even a small fraction of a volt leads to gas breakdown; the rf voltage drop across the electrodes decreases abruptly, a glow appears within the gap, a conductance current flows in the discharge circuit, and the phase shift angle between rf current and voltage becomes less than $\pi / 2$. All these changes, which appear simultaneously, are reliable indications of gas breakdown and they are easily observed. The rf voltage was varied (before the breakdown) in steps of $\Delta U_{\mathrm{rf}} \approx 0.5 \mathrm{~V}$. The gas pressure was monitored with the smallest step of $\Delta p \approx 0.001$ Torr. The rates of increase 


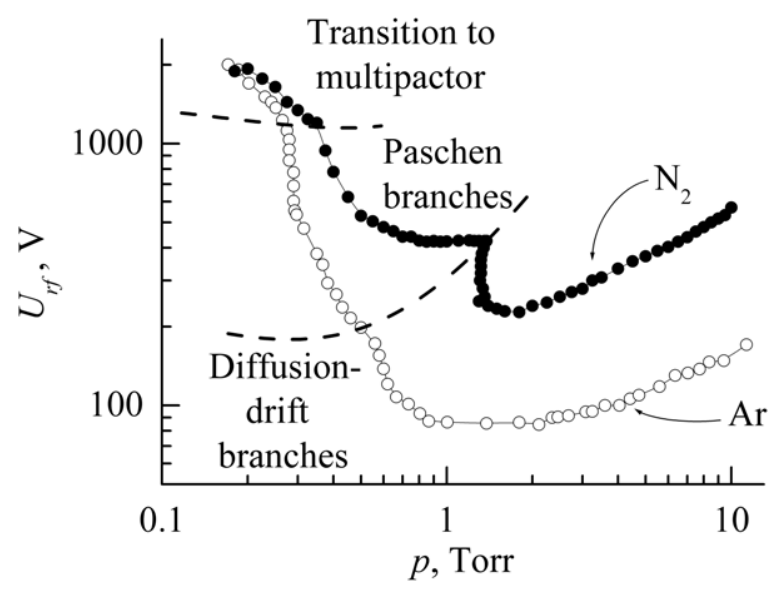

Figure 2. Rf breakdown curves in nitrogen $(L=10 \mathrm{~mm})$ and argon $(L=12 \mathrm{~mm})$.

of the pressure (while measuring to the left of rf breakdown curve minimum in the multi-valued region) and of the $\mathrm{rf}$ voltage (right-hand branch) were very small. For example, after increasing the rf voltage by a minimum step $\Delta U_{\mathrm{rf}} \approx 0.5 \mathrm{~V}$ we waited several seconds for a possible breakdown and, if not, made the following step $\Delta U_{\text {rf }}$. The spread of registered values of breakdown voltage ( $\mathrm{rf}$ as well as dc) did not exceed $1 \mathrm{~V}$.

We used the technique proposed by Levitskii [27] to measure the breakdown curves of the rf discharge. Near to, and to the high-pressure side of the minimum in the breakdown curve the gas pressure was fixed before slowly increasing the rf voltage until gas breakdown occurred. To the low-pressure side of the minimum the curve may be multi-valued, i.e. the curve turns back to higher pressures, and breakdown can occur at two different values of the rf voltage. Therefore in this range we first decreased the gas pressure, then fixed the rf voltage value and only then increased the gas pressure slowly until discharge ignition occurred.

The residence time for atoms and molecules in our chamber within the entire range of gas pressures studied was less than $1 \mathrm{~min}$, as the gas was renovated continuously. The time between successive breakdowns in our chamber was not less than $5 \mathrm{~min}$; therefore, the excited atoms and molecules after previous breakdown were swept out of the chamber and did not affect the next breakdown.

We did not apply additional sources of primary electrons initiating breakdown (UV lamps, radioactive materials, etc). Primary electrons were generated within the inter-electrode gap due to ionization of gas molecules by cosmic and terrestrial radioactive radiation.

\section{Experimental results and discussion}

As mentioned above, [9] suggested distinguishing three branches in the rf breakdown curves: diffusion-drift, Paschen and multipactor ones. Figure 2 presents the rf breakdown curves for argon and nitrogen where we also mark the branches outlined here. Under conditions of the diffusion-drift branch in the rf gas breakdown gas ionization via electron impact, electron escape to electrodes due to drift oscillations in the rf field, electron diffusion loss as well as electron induced secondary emission occur. For rf breakdown under conditions of the Paschen branch electron loss due to diffusion becomes negligibly small compared with the drift escape. Molecule ionization and electron induced secondary emission also play an important role. The multipactor branch is described by the constancy of rf breakdown voltage under gas pressure variation [28]. For igniting a multipactor discharge one has to ensure that the electron-induced secondary emission coefficient is $\delta \geqslant 1$. Secondary electrons must emerge at the moment of advantageous direction of the rf electric field (leading them away from the electrode surface), approach the opposite electrode with a sufficiently large energy (where $\delta \geqslant 1$ ) again at the moment when the rf field is small or directed to the electrode [29]. Other processes (ionization, diffusion) do not play an essential role here.

Similarly to the three modes of longitudinal combined discharge burning [25], we separate ignition of a combined discharge into three modes: (1) ignition of an rf discharge perturbed with a dc electric field; (2) ignition in combined fields; (3) ignition of a dc discharge perturbed with an rf electric field. The first ignition mode is characterized by the increase in the breakdown rf voltage under application of the dc electric field. The dc field does not contribute essentially to the ionization of gas molecules by electrons, but it increases the loss of charged particles from the inter-electrode gap due to drift in the dc field. In the second mode both $\mathrm{rf}$ and $\mathrm{dc}$ electric fields (comparable in magnitude) make a remarkable contribution to ionization. In the third mode the applied rf voltage weakly eases the dc self-sustained dc discharge. Figure 3 depicts the breakdown curves of a combined discharge in nitrogen registered at different fixed values of the dc voltage. The rf breakdown curve of the discharge $\left(U_{\mathrm{dc}}=0\right)$ with an inter-electrode distance of $L=10 \mathrm{~mm}$ possesses a region of multi-valued dependence of breakdown voltage on gas pressure [9]. But the application of even a moderate dc voltage $\left(U_{\mathrm{dc}}=50 \mathrm{~V}\right)$ leads to the disappearance of this region. In addition, increasing the dc voltage leads to an increase in the breakdown voltage at the diffusion-drift branch of the breakdown curve of the combined discharge compared with a self-sustained rf discharge. Thus we observe the combined discharge ignition in the first mode (ignition of the rf discharge perturbed by the dc electric field). Then, starting from $U_{\mathrm{dc}} \approx 250 \mathrm{~V}$, we observe a deep minimum at the pressure corresponding to the minimum in the dc discharge ignition (in figure 3 this minimum is definitely pronounced in the curve for $U_{\mathrm{dc}} \approx 300 \mathrm{~V}$ ). At such voltage values the dc electric field applied, simultaneously with an rf one, makes an essential contribution to gas ionization in the volume, thus making its breakdown easier. In this case we observe the combined discharge ignition in the second mode (ignition in combined electric fields).

At large dc voltage values $U_{\mathrm{dc}}=400$ and $600 \mathrm{~V}$ the rf breakdown curves contain the left-hand and right-hand branches (figure 3 ). These branches approach zero at pressure values when a self-sustained dc discharge ignites, and there is no necessity for applying an additional rf voltage. It is easy to explain the behaviour of the right-hand branches because 


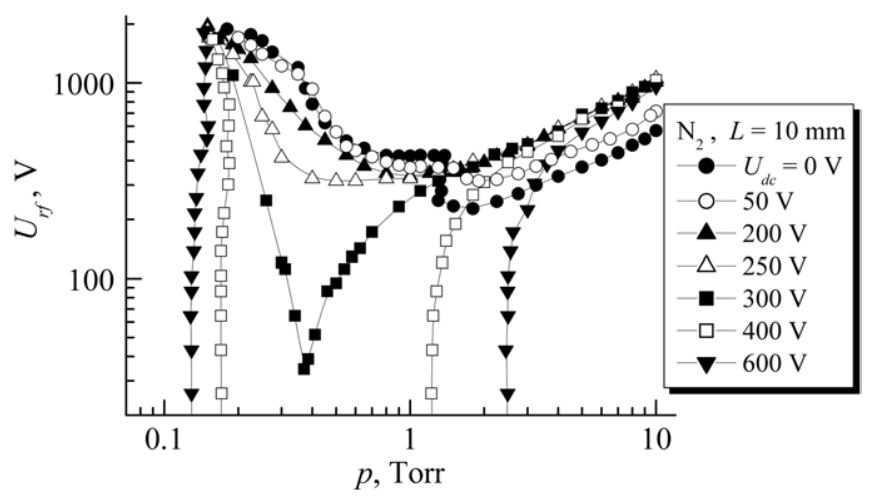

Figure 3. Breakdown curves of the combined discharge in nitrogen at different fixed values of the dc voltage, $L=10 \mathrm{~mm}$.

with the increase in the gas pressure the applied dc voltage is insufficient for breakdown. Therefore the discharge ignites after the application of the rf voltage. At small rf voltage values $U_{\text {rf }} \leqslant 100 \mathrm{~V}$ the breakdown occurs in the third mode, i.e. actually the dc discharge ignites and the rf voltage affects this process weakly. At large pressure values these branches tend to the right-hand branches of the breakdown curves for lesser dc voltage values, and the breakdown occurs in the second mode when both ( $\mathrm{rf}$ and dc) electric fields affect the ionization rate of gas molecules by electrons considerably. Now consider the left-hand side branches at large dc voltage values. These branches at $U_{\text {rf }} \leqslant 120 \mathrm{~V}$ are vertical lines, and the applied rf voltage weakly affects the dc gas breakdown (occurring in the third mode). At higher rf voltage values these branches deviate slightly to the higher gas pressure values. Perhaps under these conditions a sufficiently large rf voltage starts to impede the ionization process. During one half of the rf field period, when rf and dc fields have the same direction, particle generation due to ionization increases. However during the second half of the rf field period, the rf field weakens the dc one (down to zero at $U_{\mathrm{dc}} \approx U_{\mathrm{rf}}$ ). With narrow gap and low pressure during this halfperiod of the rf field charged particle loss is not compensated by weakened ionization. Therefore, the discharge ignites at higher pressure values when the ionization rate is higher and the electron loss due to diffusion and drift to electrodes is lower. At the rf voltage exceeding $U_{\mathrm{dc}}$ remarkably, the ionization rate remains sufficiently high during both half-periods of the rf field, and the gas breakdown may occur at lower gas pressure values (what we observe in figure 3 for $U_{\mathrm{dc}}=400$ and $600 \mathrm{~V}$ at low pressure values $p \approx 0.1-0.2$ Torr and $U_{\text {rf }} \approx 1000 \mathrm{~V}$ ). The rf breakdown curve behaviour described here is most obviously pronounced at large dc voltage values. On registering these branches we first pumped out the gas to $p \leqslant 0.1$ Torr, then we established fixed values of $\mathrm{rf}$ and dc voltages and slowly increased the gas pressure up to the moment of discharge breakdown.

In a discharge in nitrogen we observed the increase in $\mathrm{rf}$ breakdown voltage with dc voltage increasing in the pressure range $p \geqslant 1.4$ Torr (see figure 4), which corresponds to the diffusion-drift branch on the self-sustained rf discharge breakdown curve (see figures 2 and 3 ). Figure 5 shows the breakdown curves registered for the combined discharge in argon at different fixed dc voltage values in a discharge

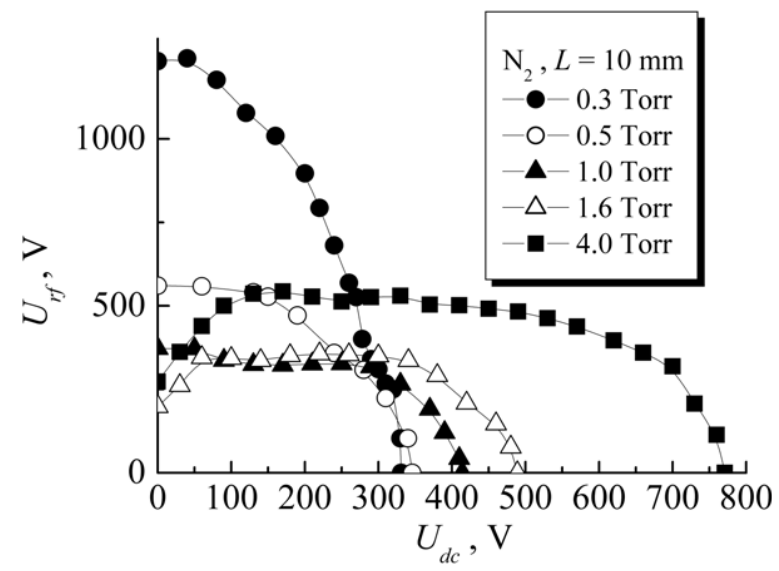

Figure 4. Rf breakdown voltage against applied dc voltage of the combined discharge at different fixed values of nitrogen pressure, $L=10 \mathrm{~mm}$.

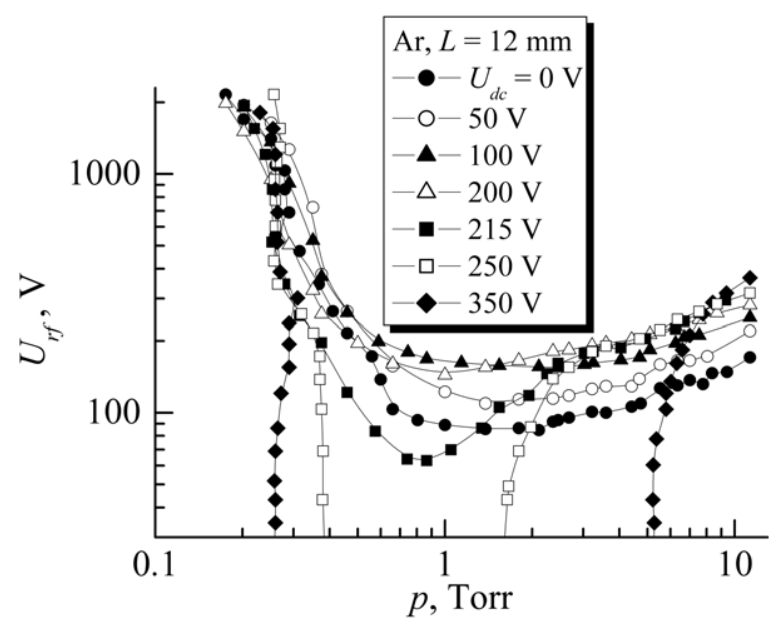

Figure 5. Breakdown curves of the combined discharge in argon at different fixed values of the dc voltage, $L=12 \mathrm{~mm}$.

chamber with the inter-electrode distance of $L=12 \mathrm{~mm}$. Under such conditions the diffusion-drift branch is most pronounced whereas the Paschen branch exists in a rather narrow argon pressure range $p=0.12-0.5$ Torr.

For a discharge in argon the increase in the rf breakdown voltage with dc voltage growth is observed over almost the entire pressure range studied in this paper (see figure 6). The presence of the multi-valued region in the rf breakdown curve in nitrogen and its absence in argon with narrow gaps are associated probably with the following circumstance. For argon the first Townsend coefficient $\alpha$ is remarkably larger than for nitrogen (particularly in the range of the reduced electric field $E / p<1000 \mathrm{~V} \mathrm{~cm}^{-1} \mathrm{Torr}^{-1}$ ) [15]. This leads to a higher rate of argon atom ionization via electron impact increasing the electron concentration within the inter-electrode gap. In addition, the longitudinal diffusion coefficient for electrons in argon exceeds that in nitrogen [30,31], which causes the spread of electron cloud in argon. Consequently, an enhanced number of particles are left inside the discharge gap providing a sufficient level of ionization under gas breakdown at lowered pressures. Therefore, the breakdown curve of the rf discharge in argon does not possess a multi-valued region for 


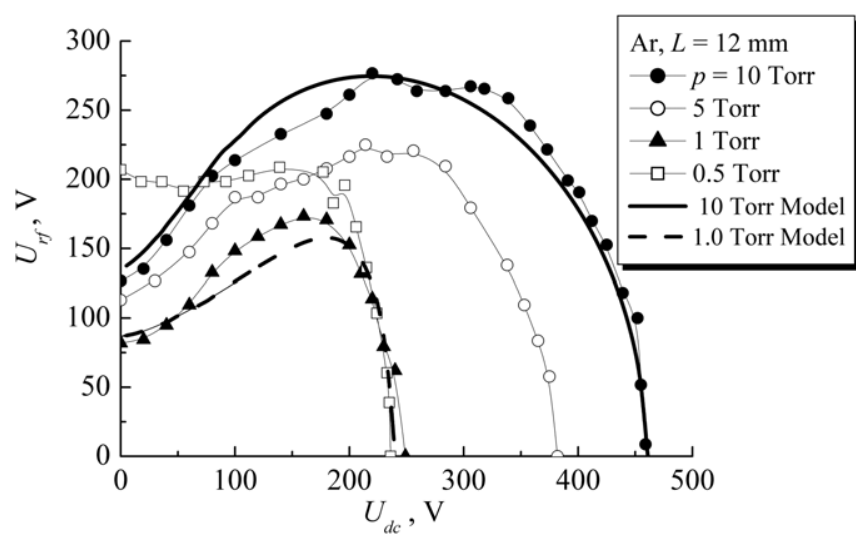

Figure 6. Rf breakdown voltage against applied dc voltage of the combined discharge at different fixed values of argon pressure, $L=12 \mathrm{~mm}$. Solid and dashed curves depict the results calculated with criterion (28) for the argon pressure values of 10 Torr and 1 Torr, respectively.

small inter-electrode gap values [9], in contrast to the discharge ignition in nitrogen. If we apply an additional dc electric field simultaneously with the rf one, electron losses increase due to the increased constant flow of electrons directed to the 'anode'. At the same time, a small dc field does not make a noticeable contribution to the ionization of gas molecules. In this case we need to apply a larger rf voltage for combined discharge ignition in the first mode than for igniting a self-sustained rf discharge. In figure 6 this effect can be observed in the pressure range $p \geqslant 0.5$ Torr. Here we observe the ignition of the combined discharge in the first mode ( $\mathrm{rf}$ discharge perturbed with dc field). When sufficiently large values of dc voltage are applied across the discharge gap, the dc field makes a considerable contribution in supporting a sufficient level of ionization for the breakdown to occur. Moreover, this level of ionization is also supported by ion-induced secondary emission. The rf breakdown voltage decreases abruptly with dc voltage growing until a self-sustained dc discharge ignites. Here we observe the combined discharge ignition in the third mode: dc discharge ignition perturbed by the rf electric field. At the dc voltage close to the minimum breakdown voltage of the dc discharge, it is necessary to apply a small rf voltage for dc discharge ignition. For example, a sharp minimum in the rf breakdown curve at $U_{\mathrm{dc}}=300 \mathrm{~V}$ is observed at the pressure $p_{\text {min }} \approx 0.36$ Torr and rf voltage $U_{\mathrm{rf}} \approx 30 \mathrm{~V}$. The minimum of the dc breakdown curve (figure $6, U_{\mathrm{rf}}=0$ ) is located at the same nitrogen pressure and $U_{\text {dc. } \min }=317 \mathrm{~V}$. With a joint action of the $\mathrm{rf}$ and dc electric fields ionization occurs in the effective electric field:

$$
E_{\mathrm{eff}}=\sqrt{E_{\mathrm{dc}}^{2}+\frac{E_{\mathrm{rf}}^{2}}{2} \cdot \frac{v_{\mathrm{en}}^{2}}{v_{\mathrm{en}}^{2}+\omega^{2}}} .
$$

Under conditions of a sharp minimum in figure $2\left(U_{\mathrm{dc}}=\right.$ $300 \mathrm{~V}$ ) we lack only $U_{\mathrm{dc}}=17 \mathrm{~V}$ for the ignition of the selfsustained dc discharge. This lacking dc voltage is compensated with the rf voltage. If we leave the minimum (to larger or smaller gas pressure values) the breakdown dc voltage increases, therefore we have to apply a higher rf voltage to ignite a combined discharge.
We consider it most interesting to study the ignition of the combined discharge in the second mode (combined discharge) when rf and dc voltages make comparably equal contributions to the ionization of gas molecules. In figure 6 the second mode for breakdown in argon is observed near the maxima of $U_{\mathrm{rf}}\left(U_{\mathrm{dc}}\right)$ curves. In figure 4 the second mode for breakdown in nitrogen corresponds to the horizontal sections of $U_{\mathrm{rf}}\left(U_{\mathrm{dc}}\right)$ dependences. It is possible that the electrons do not play the main role in gas breakdown under conditions of the Paschen branch. The mechanism of the combined breakdown in nitrogen in the second mode is similar to the rf breakdown in the range of the Paschen branch minimum. The applied dc voltage would sweep the electrons out of the discharge gap and lead to the breakdown voltage increase. But we observe in figure 4 for nitrogen (as well as in figure 6 for argon at $p=0.5$ Torr), that the $U_{\mathrm{rf}}\left(U_{\mathrm{dc}}\right)$ dependence possesses a section where the application of the dc voltage does not actually affect the breakdown rf voltage value. Perhaps under such conditions associative ionization plays an important role in producing a sufficient level of ionization and combined discharge ignition. In nitrogen the following reactions may be important:

$$
\begin{aligned}
& \mathrm{N}_{2}\left(A^{3} \Sigma_{\mathrm{u}}^{+}\right)+\mathrm{N}_{2}\left(a^{\prime 1} \Sigma_{\mathrm{u}}^{-}\right) \rightarrow \mathrm{N}_{2}^{+}+\mathrm{N}_{2}+\mathrm{e}, \\
& \rightarrow \mathrm{N}_{4}^{+}+\mathrm{e}
\end{aligned}
$$

as well as

$$
\begin{aligned}
& \mathrm{N}_{2}\left(a^{\prime 1} \Sigma_{\mathrm{u}}^{-}\right)+\mathrm{N}_{2}\left(a^{\prime 1} \Sigma_{\mathrm{u}}^{-}\right) \rightarrow \mathrm{N}_{2}^{+}+\mathrm{N}_{2}+\mathrm{e}, \\
& \rightarrow \mathrm{N}_{4}^{+}+\mathrm{e} .
\end{aligned}
$$

Here $\mathrm{N}_{2}\left(A^{3} \Sigma_{\mathrm{u}}^{+}\right)$and $\mathrm{N}_{2}\left(a^{\prime 1} \Sigma_{\mathrm{u}}^{-}\right)$are the metastable states of the nitrogen molecule with energies of $6.2 \mathrm{eV}$ and $8.4 \mathrm{eV}$, respectively. Nitrogen atoms $\mathrm{N}\left({ }^{4} \mathrm{~S}\right)$ may also play an important role in supporting the ionization level sufficient for breakdown. Collisions of these atoms as well as metastable states of nitrogen molecules with electrodes and chamber walls may also cause an increase in secondary electron emission. Again, photoemission may play a substantial role in igniting the combined discharge. Clarifying the mechanisms participating in the breakdown under the Paschen branch conditions is a complicated problem. Therefore, it is of interest to perform additional experimental and theoretical research. It is hardly probable that under conditions of the Paschen branch charged particle losses due to diffusion and drift are compensated by gas molecule ionization via electron impact at the same rf voltage within the broad range of applied dc voltage values (because the rates of direct ionization by electrons, diffusion and drift losses are complicated functions of the reduced electric field $E / p$ ). Consequently, other mechanisms of charged particle generation have to take an active (if not the main) part (in the gas volume as well as on the electrode surface due to secondary emission).

Now we consider the rf field effect on the dc discharge ignition. As far as we know, the results of such studies are absent in accessible references. Figure 7 depicts the dc breakdown voltage against nitrogen pressure at different fixed values of the applied rf voltage. The application of even a moderate $\left(U_{\mathrm{rf}}=172 \mathrm{~V}\right)$ rf voltage makes the discharge ignition easier compared with the self-sustained dc discharge. 


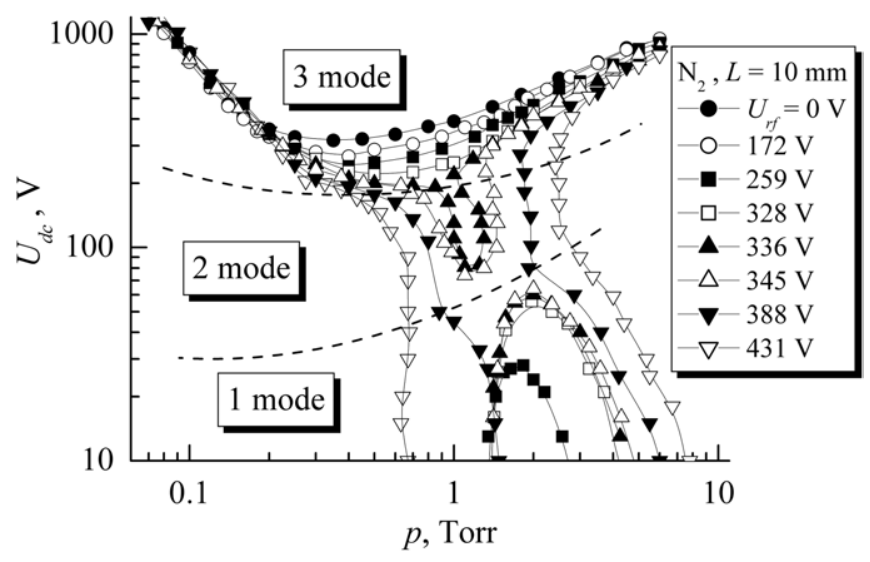

Figure 7. Dc breakdown voltage in nitrogen at different fixed if voltage values, $L=10 \mathrm{~mm}$.

In the combined electric field electrons acquire larger energy and gas ionization degree increases making the discharge ignition easier. In the range $U_{\mathrm{rf}} \leqslant 330 \mathrm{~V}$ the application of $\mathrm{rf}$ voltage simultaneously with the dc one leads to lower values of the breakdown dc voltage of the combined discharge. The breakdown curves are shifted down the ordinate axis keeping the shape similar to that of the Paschen curves (see figure 7). In this case we observe the combined discharge ignition in the third mode (ignition of the dc discharge perturbed with the rf voltage).

The breakdown curve of the dc discharge with simultaneous application of rf voltage, whose value is above the minimum voltage of the self-sustained rf discharge breakdown $U_{\text {rf.min }}$, consists of two sections. The lower section of the breakdown curve in the combined fields in figure 7 possesses the shape of an arc approaching zero values at those nitrogen pressure values when the applied rf voltage leads to the ignition of the self-sustained rf discharge. In this region the ignition of the combined discharge occurs in the first mode (rf breakdown perturbed by the dc electric field). Two values of pressure at which the combined breakdown curve approaches zero are located on both sides of the minimum voltage value on the $\mathrm{rf}$ discharge breakdown curve in nitrogen (figure 2, $U_{\text {rf. min }} \approx 230 \mathrm{~V}$ ). Within the range between these two pressure values the $\mathrm{rf}$ discharge ignites at $\mathrm{rf}$ voltages below the value $U_{\text {rf }}>U_{\text {rf. min }}$ we chose. In order to register a point at the lower branch of the breakdown curve of the combined discharge with gas pressure and rf voltage fixed we first had to apply abruptly a larger dc voltage than the ignition one and then to decrease it smoothly until the breakdown occurred. In order to register the upper branch of the curve the dc voltage after a jump to $U_{\mathrm{dc}} \approx 120 \mathrm{~V}$ (see figure 8 ) at the fixed pressure is increased slowly until the breakdown has occurred. For registering two sections of the breakdown curves in figures 7 and 8 we could also decrease the gas pressure first (down to 0.5 Torr for nitrogen and to 0.1 Torr for argon), and then establish the required value of the rf voltage and some values of dc voltage (e.g. $80 \mathrm{~V}$ for nitrogen and $120 \mathrm{~V}$ for argon). The value of the chosen dc voltage had to be between the upper and lower sections of the breakdown curve in figures 7 and 8 , respectively. After that the gas pressure was increased

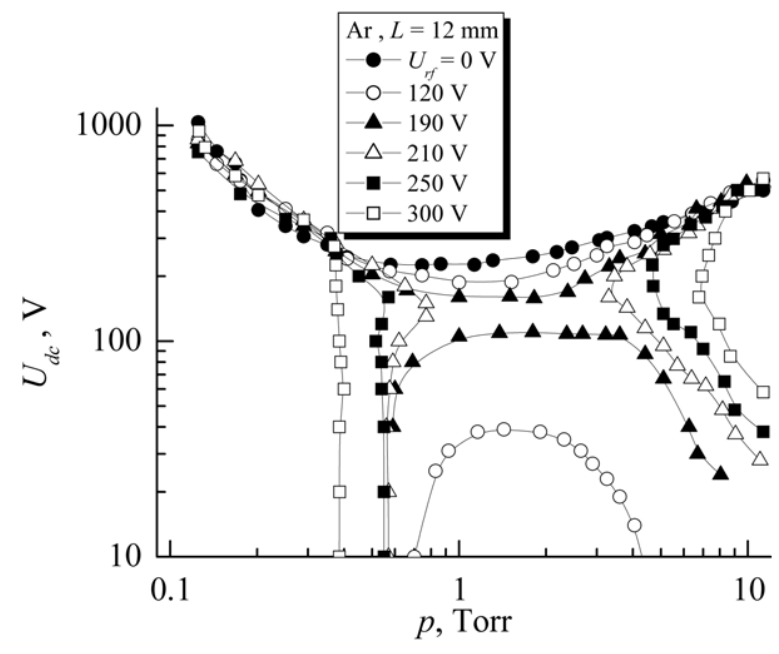

Figure 8. Dc breakdown voltage in argon at different fixed if voltage values, $L=12 \mathrm{~mm}$.

smoothly to the required fixed value. Then we were able to register the upper or lower section of the breakdown curve by increasing or decreasing the dc voltage, respectively.

The upper section of each breakdown curve for the $\mathrm{rf}$ voltage below the minimum one for the Paschen branch of the breakdown curve of the rf discharge $\left(U_{\mathrm{rf}} \approx 330 \mathrm{~V}\right)$ possesses a shape similar to the breakdown curve of the dc discharge, though it is shifted to the lower dc voltage values and higher pressure values (see figure 7). Thus, for the same rf voltage value combined with the rf one, one may observe the ignition in two modes. The upper section of the breakdown curve corresponds to the ignition in the third mode of the combined discharge whereas the lower one relates to the first mode.

Using the Townsend breakdown equation [15]

$$
\gamma\left[\mathrm{e}^{\alpha L}-1\right]=1,
$$

where $\alpha$ is the first Townsend coefficient for ionization, $\gamma$ is the ion-induced secondary electron emission coefficient, we may determine the dependence of $\gamma$ on the reduced electric field $E / p$ for nitrogen and argon. As the coefficient $\gamma$ depends not only on the gas species but also on the material and state of the cathode surface, it is expedient to determine $\gamma$ under conditions of our experiments. To this end we obtained the dependence of $\alpha / p$ on $E / p$ with the Bolsig numerical code permitting us to find the transport coefficients of electrons in a number of gases with the superimposed electric field $E$. In this paper we also used the measured and calculated data of [31-36] and obtained the approximation formulae $\alpha / p(E / p)$ for argon,

$\frac{\alpha}{p}=1.6 \times 10^{3} \cdot \exp \left[-\frac{19}{(E / p)^{0.24}}-0.021 \cdot(E / p)^{0.9}\right]$,

and nitrogen,

$\frac{\alpha}{p}=134 \cdot \exp \left[-\frac{65}{(E / p)^{0.53}}-0.0021 \cdot\left(\frac{E}{p}\right)^{0.853}\right]$.

Here $\alpha / p$ is measured in $\mathrm{cm}^{-1} \operatorname{Torr}^{-1}$, and the reduced electric field $E / p$ is measured in $\mathrm{V} \mathrm{cm}^{-1} \mathrm{Torr}^{-1}$. Using the breakdown 


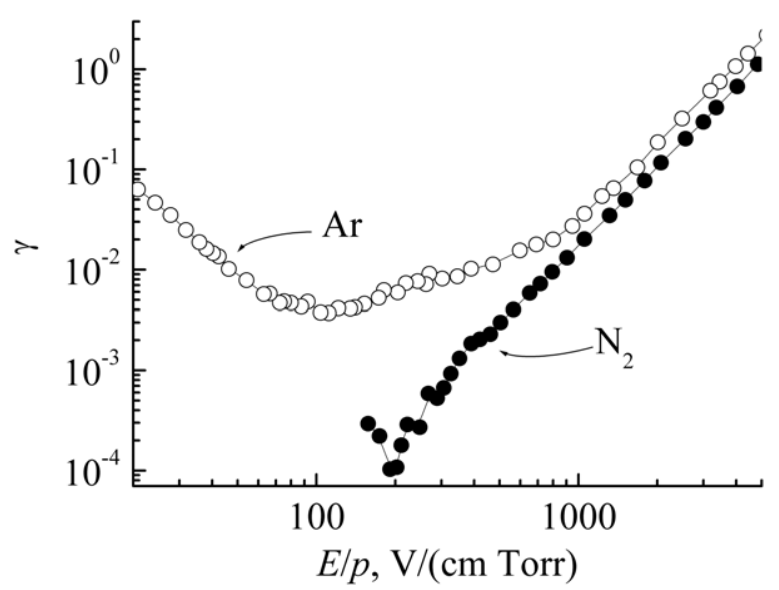

Figure 9. Ion-induced secondary electron emission coefficient against the ratio of dc electric field to gas pressure for nitrogen and argon, cathode is made out of stainless steel.

curves of the dc discharge $U_{\mathrm{dc}}(p)$ (at $U_{\mathrm{rf}}=0$ ) presented in figures 7 and 8 together with formulae (4)-(6) we determined the dependences $\gamma(E / p)$ for argon and nitrogen (see figure 9$)$. It is clear from figure 9 that the ion-induced secondary electron emission coefficient $\gamma$ from the cathode surface under bombardment with argon ions exceeds that for nitrogen ions within the total range of $E / p$ values. More intense ion-induced secondary electron emission under discharge ignition in argon leads to a transition of the combined gas breakdown from the first mode to the third one, in fact avoiding the weakly expressed second mode. The only exclusion is observed at lower pressure values when a Paschen branch is available in the breakdown curve (figure 6 , the curve for $p=0.5$ Torr). In nitrogen the transition of the combined breakdown from the second mode to the third one sets in at much higher dc voltage values $\left(U_{\mathrm{dc}}>300 \mathrm{~V}\right)$ than in $\operatorname{argon}\left(U_{\mathrm{dc}} \approx 150-300 \mathrm{~V}\right)$. The second mode of the discharge ignition in combined fields is observed when the rf voltage applied across the dc discharge approaches the value corresponding to the minimum of the Paschen branch of the self-sustained rf discharge. For this branch the minimum breakdown voltage does not depend on the discharge gap width and the frequency of the applied rf field [37]. Again, we observe in figure 4 that under conditions of the Paschen branch for nitrogen the application of the dc voltage does not practically affect the breakdown voltage of the combined discharge. Deep minima of the dc breakdown curves in figure 7 correspond to the horizontal sections of the dependences of rf breakdown voltage on the applied dc voltage in figure 4. Thus, the joint application of the dc voltage and the rf one corresponding to the minimum voltage of the Paschen branch of the rf breakdown curve in nitrogen $\left(U_{\mathrm{rf}} \approx 330 \mathrm{~V}\right)$ is accompanied by a sharp minimum in the breakdown curves of the dc discharge in the rf field (figure 7). Simultaneously, we observe the gas breakdown and combined discharge ignition in the second mode (combined discharge), when the applied rf and dc electric fields have comparable values. We do not observe deep minima in the breakdown curves of the combined discharge in argon (see figure 8). With a subsequent increase in the rf voltage $\left(U_{\mathrm{rf}}>350 \mathrm{~V}\right)$ the upper and lower sections of the breakdown curves of the combined discharge in argon as well as in nitrogen merge and they are transformed into the left and right branches (see figures 7 and 8).

Figure 8 demonstrates the breakdown curves of the combined discharge in argon for various fixed values of the rf voltage. As the minimum rf breakdown voltage under given conditions amounts to $U_{\text {rf. } \min }=85 \mathrm{~V}$, the simultaneous application of the dc voltage and the rf one $U_{\text {rf }}=120 \mathrm{~V}$ leads to the separation of the breakdown curve of the combined discharge into two sections. Similarly to the nitrogen case, the upper section of the curve corresponds to the breakdown in the third mode (dc breakdown perturbed by the rf voltage), and the lower one corresponds to the first one (rf breakdown perturbed by the dc voltage). At $U_{\mathrm{rf}} \geqslant 200 \mathrm{~V}$ the upper and lower sections of the combined breakdown curves merge.

Thus, our experimental data imply that the ignition of the combined discharge may occur in one of the three possible modes: (1) the ignition of an $\mathrm{rf}$ discharge perturbed by a dc electric field; (2) the ignition in combined fields; (3) the ignition of a dc discharge perturbed by an rf electric field. The mode in which ignition is to be observed depends on the gas species and pressure in the discharge chamber and the relation between the $\mathrm{rf}$ and dc voltages applied across the electrodes. With $\mathrm{rf}$ voltage values exceeding the minimum breakdown voltage of the $\mathrm{rf}$ discharge the breakdown curve of the combined discharge for the same fixed voltage value consists of two sections. The upper one corresponds to the ignition of the combined discharge in the third mode whereas the lower one corresponds to the ignition in the first mode. With rf voltage values exceeding the minimum one of the Paschen branch of the rf discharge breakdown curve, the application of the dc voltage leads to the ignition of the combined discharge in the second mode.

\section{Analytical model of gas breakdown in combined fields}

Let us consider a gap filled with a gas in a cylindrical chamber with height (distance between flat electrodes) equal to $L$ and radius equal to $R$. Let us direct the $z$-coordinate axis along the chamber axis with the radial coordinate $r$ directed across this axis. The origin of the coordinate set is located at the gap centre between the electrodes. We regard the rf and dc fields to be uniform throughout the gap and electron, $n_{\mathrm{e}}$, and positive ion, $n_{\mathrm{i}}$, number densities to be small and not disturbing the electric fields. We will write the balance equations for positive ions

$$
\frac{\partial n_{\mathrm{i}}}{\partial t}=v_{\mathrm{i}} n_{\mathrm{e}}+V_{\mathrm{i}} \frac{\partial n_{\mathrm{i}}}{\partial z}
$$

and electrons

$$
\begin{aligned}
\frac{\partial n_{\mathrm{e}}}{\partial t} & =v_{\mathrm{i}} n_{\mathrm{e}}+D_{\mathrm{e}} \frac{1}{r} \frac{\partial}{\partial r}\left(r \frac{\partial n_{\mathrm{e}}}{\partial r}\right)+D_{\mathrm{L}} \frac{\partial^{2} n_{\mathrm{e}}}{\partial z^{2}}-V_{\mathrm{dc}} \frac{\partial n_{\mathrm{e}}}{\partial z} \\
& -V_{\mathrm{rf}} \frac{\partial n_{\mathrm{e}}}{\partial z} \cos (\omega t),
\end{aligned}
$$

where $v_{\mathrm{i}}$ is the rate of gas molecule ionization via electron impact, $V_{\mathrm{i}}$ is the ion drift velocity in the dc electric field, $D_{\mathrm{L}}$ and $D_{\mathrm{e}}$ are the coefficients of longitudinal and transverse diffusion of electrons, respectively, $V_{\mathrm{dc}}$ and $V_{\mathrm{rf}}$ are the electron velocities 
in the dc and rf electric fields, $\omega=2 \cdot \pi \cdot f$ is the angular frequency of the $\mathrm{rf}$ field. We choose the following boundary conditions. Electron number density vanishes at the discharge tube wall (at $r=R$ ), i.e.

$$
\left.n_{\mathrm{e}}(r, z)\right|_{r=R}=0
$$

At the 'cathode' (the electrode with the negative potential located at $z=-L / 2$ ) we have

$$
\left.n_{\mathrm{e}} \cdot V_{\mathrm{dc}}\right|_{z=-L / 2}=\left.\gamma \cdot n_{\mathrm{i}} \cdot V_{\mathrm{i}}\right|_{z=-L / 2},
$$

where $\gamma$ is the ion-induced secondary electron emission coefficient for the cathode surface, whereas for the 'anode' (located at $z=L / 2$ ) we have

$$
\begin{gathered}
\left.n_{\mathrm{i}} \cdot V_{\mathrm{i}}\right|_{z=L / 2}=0, \\
\left.n_{\mathrm{e}}(r, z)\right|_{z=L / 2}=0 .
\end{gathered}
$$

Let us transform to the reference frame in which an electron oscillating in the rf electric field is at rest, i.e.

$$
z^{*}=z-\frac{V_{\mathrm{rf}}}{\omega} \cdot \sin (\omega t) .
$$

Then boundary conditions (10)-(12) assume the form

$$
\begin{gathered}
\left.n_{\mathrm{e}} \cdot V_{\mathrm{dc}}\right|_{z^{*}=-}\left(\frac{L}{2}-\frac{V_{\mathrm{rf}}}{\omega}\right) \\
\left.n_{\mathrm{i}} \cdot V_{\mathrm{i}}\right|_{z^{*}=\frac{L}{2}-\frac{V_{\mathrm{rf}}}{\omega}}=0,\left.n_{\mathrm{i}} \cdot V_{\mathrm{i}}\right|_{z^{*}=-\left(\frac{L}{2}-\frac{V_{\mathrm{rf}}}{\omega}\right)}, \\
\left.n_{\mathrm{e}}\left(r, z^{*}\right)\right|_{z^{*}=\frac{L}{2}-\frac{V_{\mathrm{rf}}}{\omega}}=0,
\end{gathered}
$$

and balance equations (7) and (8) are transformed as follows:

$$
\begin{aligned}
& \frac{\partial n_{\mathrm{i}}}{\partial t}=v_{\mathrm{i}} \cdot n_{\mathrm{e}}+V_{\mathrm{i}} \cdot \frac{\partial n_{\mathrm{i}}}{\partial z^{*}}, \\
& \frac{\partial n_{\mathrm{e}}}{\partial t}=v_{\mathrm{i}} \cdot n_{\mathrm{e}}+D_{\mathrm{e}} \cdot \frac{1}{r} \frac{\partial}{\partial r}\left(r \frac{\partial n_{\mathrm{e}}}{\partial r}\right)+D_{\mathrm{L}} \cdot \frac{\partial^{2} n_{\mathrm{e}}}{\partial z^{* 2}}-V_{\mathrm{dc}} \cdot \frac{\partial n_{\mathrm{e}}}{\partial z^{*}} .
\end{aligned}
$$

We will seek the stationary solution of equations (17) and (18), i.e. with $\partial n_{\mathrm{e}} / \partial t=0$ and $\partial n_{\mathrm{i}} / \partial t=0$. Then equation (18) can be rewritten as

$$
\begin{aligned}
\frac{v_{\mathrm{i}}}{D_{\mathrm{e}}}+ & \frac{1}{n_{\mathrm{e}}} \cdot \frac{1}{r} \frac{\partial}{\partial r}\left(r \frac{\partial n_{\mathrm{e}}}{\partial r}\right)+\frac{D_{\mathrm{L}}}{D_{\mathrm{e}}} \cdot \frac{1}{n_{\mathrm{e}}} \cdot \frac{\partial^{2} n_{\mathrm{e}}}{\partial z^{* 2}}-\frac{V_{\mathrm{dc}}}{D_{\mathrm{e}}} \\
& \frac{1}{n_{\mathrm{e}}} \cdot \frac{\partial n_{\mathrm{e}}}{\partial z^{*}}=0 .
\end{aligned}
$$

Obviously, each of the terms possesses the same dimension $\left(\mathrm{cm}^{-2}\right)$, the second term being dependent only on $r$, whereas the third and the fourth ones depend only on $z^{*}$. Therefore equation (19) can be separated into two equations:

$$
\begin{gathered}
\frac{v_{\mathrm{i}}}{D_{\mathrm{e}}}+\frac{1}{n_{\mathrm{e}}} \cdot \frac{1}{r} \frac{\partial}{\partial r}\left(r \frac{\partial n_{\mathrm{e}}}{\partial r}\right)-C^{2}=0, \\
\frac{D_{\mathrm{L}}}{D_{\mathrm{e}}} \cdot \frac{1}{n_{\mathrm{e}}} \cdot \frac{\partial^{2} n_{\mathrm{e}}}{\partial z^{* 2}}-\frac{V_{\mathrm{dc}}}{D_{\mathrm{e}}} \cdot \frac{1}{n_{\mathrm{e}}} \cdot \frac{\partial n_{\mathrm{e}}}{\partial z^{*}}=-C^{2} .
\end{gathered}
$$

It is easy to solve (20) and (21) through the separation of variables $r$ and $z^{*}$. Then for the number densities of electrons we get the solution

$$
\begin{aligned}
& n_{\mathrm{e}}\left(z^{*}, r\right)=A \cdot \exp \left(b z^{*}\right) \cdot\left[C_{1} \cdot \cos \left(a z^{*}\right)+C_{2} \cdot \sin \left(a z^{*}\right)\right] \\
& \quad \cdot J_{0}\left(\frac{2.4}{R} r\right),
\end{aligned}
$$

where $J_{0}$ is the Bessel function of zero order. It also follows from (20) that

$$
C^{2}=\frac{\nu_{\mathrm{i}}}{D_{\mathrm{e}}}-\left(\frac{2.4}{R}\right)^{2} .
$$

In equation (22) we make the following notation:

$$
\begin{gathered}
b=\frac{V_{\mathrm{dc}}}{2 \cdot D_{\mathrm{L}}}, \\
a=\sqrt{\frac{\nu_{\mathrm{i}}}{D_{\mathrm{L}}}-\frac{D_{\mathrm{e}}}{D_{\mathrm{L}}} \cdot\left(\frac{2.4}{R}\right)^{2}-\frac{V_{\mathrm{dc}}^{2}}{4 \cdot D_{\mathrm{L}}^{2}}} .
\end{gathered}
$$

Applying boundary condition (16), we rewrite the equation for the electron number density in the form

$n_{\mathrm{e}}\left(z^{*}, r\right)=C_{2} \cdot A \cdot \exp \left(b z^{*}\right)$

$$
\begin{aligned}
& \cdot\left\{\left[\sin \left(a z^{*}\right)-\cos \left(a z^{*}\right) \cdot \tan \left[a\left(\frac{L}{2}-\frac{V_{\mathrm{rf}}}{\omega}\right)\right]\right]\right\} \\
& \cdot J_{0}\left(\frac{2.4}{R} r\right) .
\end{aligned}
$$

Inserting (26) into the equation for ion balance (17) (with $\left.\partial n_{\mathrm{i}} / \partial t=0\right)$, we obtain, taking account of the boundary condition for ions at the 'anode' (15), the following equation for the ion number density:

$$
\begin{aligned}
& n_{\mathrm{i}}\left(z^{*}, r\right)=C_{2} \cdot A \cdot \frac{v_{\mathrm{i}}}{V_{\mathrm{i}}} \cdot \frac{1}{a^{2}+b^{2}} \cdot\left\{\exp \left[b \cdot\left(\frac{L}{2}-\frac{V_{\mathrm{rf}}}{\omega}\right)\right]\right. \\
& \quad\left\{b \cdot \sin \left[a \cdot\left(\frac{L}{2}-\frac{V_{\mathrm{rf}}}{\omega}\right)\right]-a \cdot \cos \left[a \cdot\left(\frac{L}{2}-\frac{V_{\mathrm{rf}}}{\omega}\right)\right]\right\} \\
& \quad-\tan \left[a\left(\frac{L}{2}-\frac{V_{\mathrm{rf}}}{\omega}\right)\right] \cdot\left\{b \cdot \cos \left[a\left(\frac{L}{2}-\frac{V_{\mathrm{rf}}}{\omega}\right)\right]\right. \\
& \left.\quad+a \cdot \sin \left[a\left(\frac{L}{2}-\frac{V_{\mathrm{rf}}}{\omega}\right)\right]\right\}-\exp \left(b \cdot z^{*}\right) \cdot\left\{b \cdot \sin \left(a z^{*}\right)\right. \\
& \quad-a \cdot \cos \left(a z^{*}\right)-\tan \left[a\left(\frac{L}{2}-\frac{V_{\mathrm{rf}}}{\omega}\right)\right] \\
& \left.\left.\quad\left[b \cdot \cos \left(a z^{*}\right)+a \cdot \sin \left(a z^{*}\right)\right]\right\}\right\} \cdot J_{0}\left(\frac{2.4}{R} r\right) .
\end{aligned}
$$

Inserting (26) and (27) into the boundary condition at the 'cathode' (14) and making simple transformations we get the generalized gas breakdown criterion in the longitudinal combined electric field for arbitrary values of $\mathrm{rf}$ and dc fields:

$$
\begin{aligned}
1= & \frac{\gamma \cdot v_{\mathrm{i}}}{2 D_{\mathrm{L}} \cdot R_{1}} \cdot\left\{\frac{2 D_{L} \cdot a}{V_{\mathrm{dc}} \cdot \sin \left[2 a \cdot\left(\frac{L}{2}-\frac{V_{\mathrm{rf}}}{\omega}\right)\right]}\right. \\
& \cdot\left\{\exp \left[\frac{V_{\mathrm{dc}}}{D_{\mathrm{L}}} \cdot\left(\frac{L}{2}-\frac{V_{\mathrm{rf}}}{\omega}\right)\right]\right. \\
& \left.\left.-\cos \left[2 a \cdot\left(\frac{L}{2}-\frac{V_{\mathrm{rf}}}{\omega}\right)\right]\right\}-1\right\},
\end{aligned}
$$


where the following notation is used

$$
R_{1}=\frac{v_{\mathrm{i}}}{D_{\mathrm{L}}}-\frac{D_{\mathrm{e}}}{D_{\mathrm{L}}} \cdot\left(\frac{2.4}{R}\right)^{2}
$$

In the absence of the dc electric field $\left(V_{\mathrm{dc}}=0\right)$ criterion (28) reduces to the rf gas breakdown criterion $[9,38]$ :

$$
\frac{\nu_{\mathrm{i}}}{D_{\mathrm{L}}}=\frac{D_{\mathrm{e}}}{D_{\mathrm{L}}} \cdot\left(\frac{2.4}{R}\right)^{2}+\left(\frac{\pi}{(L / 2)-\left(V_{\mathrm{rf}} / \omega\right)}\right)^{2} .
$$

In the following numerical calculations for argon we assumed the gas molecule ionization as well as longitudinal and transverse electron diffusion to be determined by the effective electric field (1). For the first Townsend coefficient for argon ionization we used formula (5) (substituting $E_{\text {eff }}$ instead of $E$ ). For drift velocities of electrons in $\mathrm{rf}$ and $\mathrm{dc}$ electric fields ( $V_{\mathrm{rf}}$ and $V_{\mathrm{dc}}$, respectively), as well as for the coefficients of transverse $D_{\mathrm{e}}$ and longitudinal $D_{\mathrm{L}}$ diffusion of electrons we employed the following approximate formulae (also obtained with the Bolsig numerical code):

$V_{\mathrm{rf}}=\frac{E_{\mathrm{rf}}}{p} \cdot 4 \times 10^{5} \cdot\left[8+1.13 \times 10^{-3} \cdot\left(\frac{E_{\mathrm{rf}}}{p}\right)^{1.5}\right]^{-0.3}$,

$V_{\mathrm{dc}}=\frac{E_{\mathrm{dc}}}{p} \cdot 4 \times 10^{5} \cdot\left[8+1.13 \times 10^{-3} \cdot\left(\frac{E_{\mathrm{dc}}}{p}\right)^{1.5}\right]^{-0.3}$,

$D_{\mathrm{e}}=\frac{1}{p} \cdot\left[2 \times 10^{6}+13.1 \cdot\left(\frac{E_{\mathrm{eff}}}{p}\right)^{1.8}\right]$

$D_{\mathrm{L}}=\frac{1}{p} \cdot\left[6 \times 10^{5} \cdot\left(\frac{E_{\mathrm{eff}}}{p}\right)^{-0.21}+1.31 \times 10^{5} \cdot\left(\frac{E_{\mathrm{eff}}}{p}\right)^{0.43}\right]$.

Figure 10 shows the dc breakdown voltage values at different fixed $\mathrm{rf}$ voltage values registered in argon at $L=32 \mathrm{~mm}$, as well as that calculated from criterion (28). The figure demonstrates satisfactory agreement between our experimental and calculated data. At a sufficiently large rf voltage, when a self-sustained rf discharge can be ignited, breakdown curves $U_{\mathrm{dc}}(p)$ possess two sections (conventional, U-shaped upper one, as well as a new arc-shaped lower one discovered by us). With rf voltage growing, these two sections merge and are transformed to the left (from the low-pressure side) and right (from the high-pressure side) branches.

The same criterion (28) helps us to study the effect of the applied dc voltage on breakdown rf voltage. Figure 6 shows good agreement between the calculation results got according to criterion (28) and experimental data obtained for a narrow inter-electrode gap $(L=12 \mathrm{~mm})$. Figures 11 and 12 depict the cases of low dc voltage $\left(U_{\mathrm{dc}} \leqslant 100 \mathrm{~V}\right)$ and high dc voltage $\left(U_{\mathrm{dc}} \geqslant 150 \mathrm{~V}\right)$ for a gap $L=32 \mathrm{~mm}$. In the first case the dc voltage leads to an enhanced escape of electrons to the 'anode', not contributing remarkably to the ionization rate of gas molecules via electrons. The rf breakdown curves

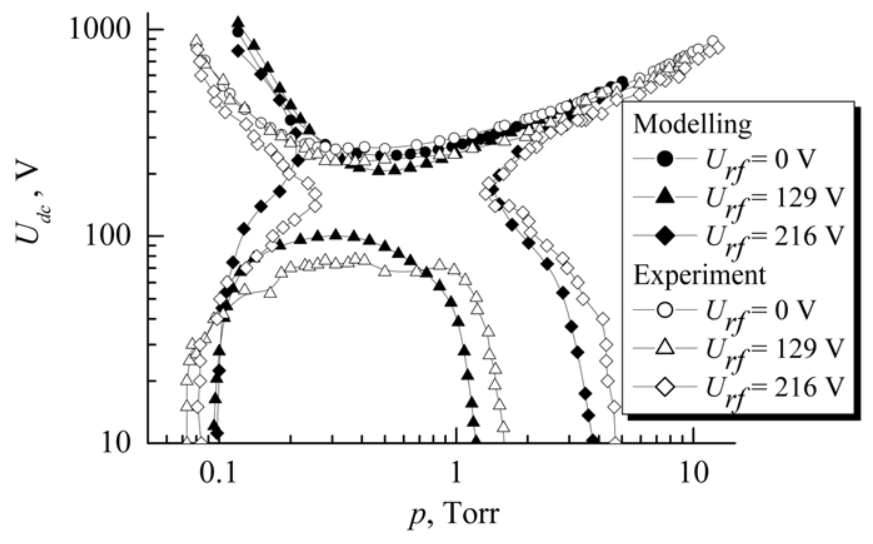

Figure 10. Dc breakdown voltage in argon registered at different fixed rf voltage values as well as that calculated from criterion (28), $L=32 \mathrm{~mm}$.

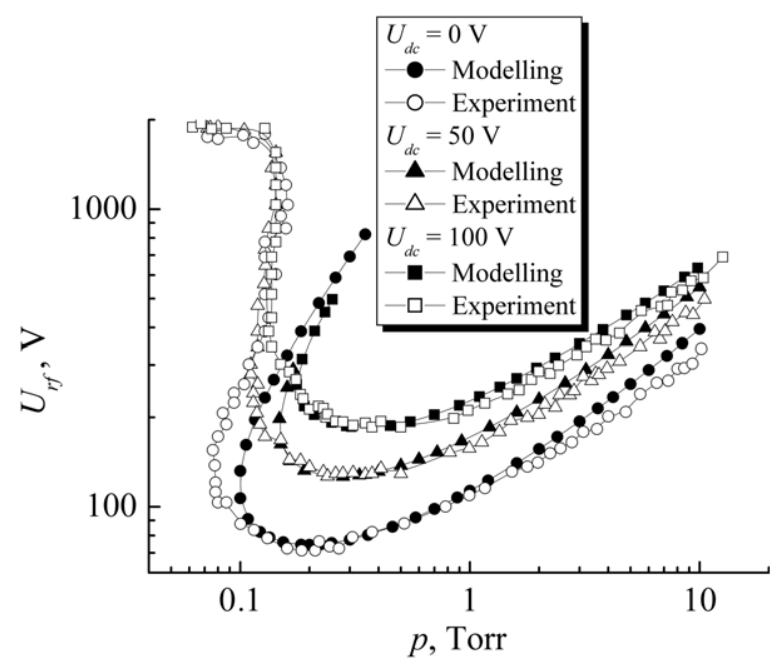

Figure 11. Rf breakdown voltage in argon registered at dc voltage values $U_{\mathrm{dc}}=0 \mathrm{~V}, 50 \mathrm{~V}$ and $100 \mathrm{~V}$, as well as that calculated from criterion (28), $L=32 \mathrm{~mm}$.

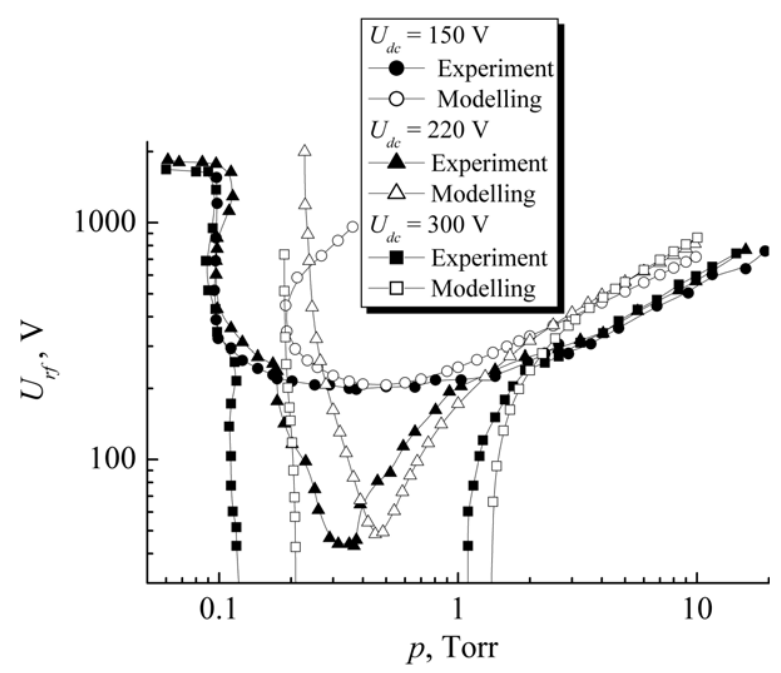

Figure 12. Rf breakdown voltage in argon registered at fixed dc voltage values $U_{\mathrm{dc}}=150 \mathrm{~V}, 220 \mathrm{~V}$ and $300 \mathrm{~V}$, as well as that calculated from criterion (28), $L=32 \mathrm{~mm}$. 
obtained with criterion (28) have the shape of a tilted parabola and furnish a good description of the diffusion-drift branch. The variation of the ion-induced secondary electron emission coefficient $\gamma$ actually does not affect the rf breakdown voltage values. Therefore, the application of complicated models [24] for secondary emission is not justified in this case. Increasing the dc voltage shifts the 'parabolas' to higher breakdown voltage and pressure values. The experimental rf breakdown curves possess at low pressures a section of the Paschen branch on which at large $L$ we observe an rf breakdown voltage growth with the gas pressure practically unchanged. It is clear from figure 11 that the Paschen branches at different $U_{\mathrm{dc}}$ are superimposed on each other indicating the weak role of electrons in the gas breakdown under these conditions (we discussed this in the above text). Again, it follows from figure 11 (and from figure 12) that multipactor branches happen to depend weakly on $U_{\mathrm{dc}}$ variation which needs further study. Discrepancy between the calculated and registered data for the Paschen and multipactor branches indicates that some processes take part in the gas breakdown not accounted for by our model.

At higher $U_{\mathrm{dc}}$ values (figure 12) the 'parabola' starts to open (its upper section at $U_{\mathrm{dc}}=150 \mathrm{~V}$ runs above the upper sections for lower $U_{\mathrm{dc}}$ values). At $U_{\mathrm{dc}}=220 \mathrm{~V}$ the region of multi-valued dependence of the rf breakdown voltage on gas pressure is already absent in the model curve, but a deep minimum is observed located at the argon pressure close to the minimum on the dc breakdown curve (see figure 10). With subsequent $U_{\mathrm{dc}}$ increase a self-sustained dc discharge ignites in a certain range of gas pressure values, therefore the rf breakdown voltage falls to zero at the boundaries of this region. Accordingly, the breakdown curves possess the left and right branches.

The left-hand branch of the breakdown curve in figure 12, obtained from criterion (28), is almost a vertical line with a weak tilt to the lower gas pressure values. At the same time, the registered rf breakdown curves at low and high $U_{\mathrm{dc}}$ values possess a multi-valued region originating from the Paschen branch and shifting to higher rf breakdown voltage and lower pressure values. This multi-valued region was discovered in [25], where the authors assumed that ionization under these conditions occurs during the part of the rf field half-period when the rf and dc electric fields possess opposite directions. Let us check the validity of this assumption.

Let us write the equation of electron motion in the combined field:

$m \frac{\mathrm{d}^{2} z}{\mathrm{~d} t^{2}}=-e \cdot\left(E_{\mathrm{dc}}+E_{\mathrm{rf}} \cdot \cos (\omega t)\right)-m \cdot v_{m} \cdot \frac{\mathrm{d} z}{\mathrm{~d} t}$,

where $e$ and $m$ are the electron charge and mass, respectively, $z$ is the axial coordinate $(z=0 \mathrm{~mm}$ at the 'cathode' and $z=32 \mathrm{~mm}$ at the 'anode'), $v_{m}$ is the rate of electron-neutral collisions. Let us perform calculations at the argon pressure of $p=0.09$ Torr, $U_{\mathrm{dc}}=300 \mathrm{~V}$ and $U_{\mathrm{rf}}=500 \mathrm{~V}$. Under these conditions the rf breakdown curve in figure 12 possesses the turning point. With the help of equation (35) we obtain (see figure 13) that the electrons generated near the 'cathode' surface (due to ionization or secondary emission) at the moment $\omega t \approx 1.2 \mathrm{rad}$ are first accelerated towards the 'anode'

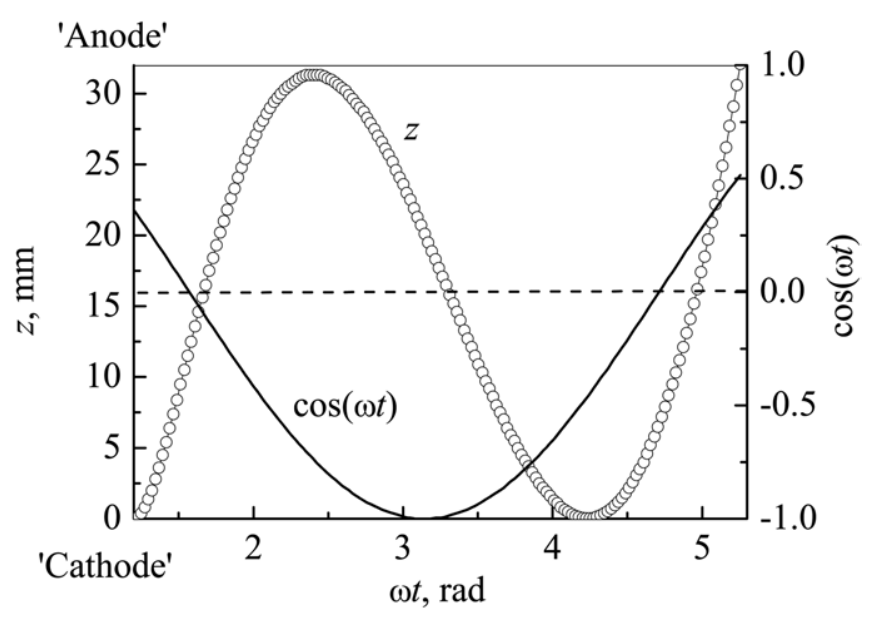

Figure 13. Trajectories of electron motion in the inter-electrode gap as well as the graph of the function $\cos (\omega t)$; argon, $p=0.09$ Torr, $U_{\mathrm{dc}}=300 \mathrm{~V}$ and $U_{\mathrm{rf}}=500 \mathrm{~V}$.

(ionizing gas molecules on their way). Then, after $\omega t=\pi / 2$, the rf field changes its sign, the resulting electric field becomes weaker and then changes its sign. This decelerates electrons that do not reach the 'anode' and stop near its surface and are then accelerated towards the 'cathode'. However, now they move comparatively slowly in a weaker resulting electric field (because rf and dc fields possess different signs), but still sufficient to support ionization, and electron drift losses decrease. Then the rf field value decreases again, electrons are slowed down, they stop at the 'cathode' and after that gain their velocity and are lost at the 'anode'. Thus at low gas pressures the conditions exist under which the electrons are capable of crossing the discharge gap thrice (not being lost on electrodes during more than the rf field half-period), performing ionizing collisions on their way. Also, those electrons that are generated due to ionization may have a long life, which increases the probability of ionizing collisions and shifts the rf breakdown curve to lower pressure values.

\section{Conclusions}

This paper studies in experiment and theory the ignition of a longitudinal combined $(\mathrm{rf}+\mathrm{dc})$ discharge in argon and nitrogen. Special attention is paid to gas breakdown in narrow gaps $(L \sim 10 \mathrm{~mm}$ ) because with narrow gaps the $\mathrm{rf}$ breakdown curves possess not only the diffusion-drift, but also the Paschen branches. We show that the combined discharge ignition may be separated into three modes: (1) rf discharge ignition perturbed by the dc electric field; (2) ignition in combined fields; (3) dc discharge ignition perturbed by the rf electric field. In the first mode the application of a weak dc voltage increases the rf breakdown voltage, in addition the dc voltage makes no remarkable contribution to gas molecule ionization via electron impact, but leads to enhanced drift loss of charged particles to electrodes. In the second mode, at higher dc voltages, rf as well as dc electric fields impart considerable energy to electrons and make the ionization process easier. With the subsequent growth of the $\mathrm{dc}$ voltage the rf breakdown voltage approaches its maximum 
and then decreases and approaches zero when the dc voltage becomes sufficient for igniting the self-sustained dc discharge. In the third mode of breakdown a weak rf field makes the dc discharge ignition easier, so that the gas breakdown occurs at lower dc voltage values than are necessary for igniting a selfsustained dc discharge. The influence of the rf voltage on dc gas breakdown was not studied in detail earlier. Application of the rf voltage exceeding the minimum breakdown voltage of the self-sustained rf discharge results in the fact that the dependence of the dc breakdown voltage on gas pressure in the chamber consists of two sections for one and the same value of the rf voltage. Our analytical model furnishes the gas breakdown criterion in the longitudinal combined electric field describing the effect of the dc field on the rf gas breakdown as well as that of the rf field on the dc gas breakdown. The calculation data agree with registered ones satisfactorily.

\section{References}

[1] Lai W T, Hwang C J, Wang A T, Yau J C, Liao J H, Chen L H, Adachi K and Okamoto S 2006 Proc. Int. Symp. on Dry Process (Nagoya, Japan) (Institute of Electrical Engineers) p 109

[2] Kawamura E, Lieberman M A, Lichtenberg A J and Hudson E A 2007 J. Vac. Sci. Technol. A 251456

[3] Eckbreth A C and Davis J W 1972 Appl. Phys. Lett. 2125

[4] Brown C O and Davis J W 1972 Appl. Phys. Lett. 21480

[5] Denpoh K and Ventzek P L G 2008 J. Vac. Sci. Technol. A 261415

[6] Rakhimova T V and Rakhimov A T 1975 Sov. J. Plasma Phys. 1468

[7] Myshenkov V I and Yatsenko N A 1982 Sov. J. Plasma Phys. 8 397

[8] Lisovskiy V, Booth J P, Landry K, Douai D, Cassagne V and Yegorenkov V 2008 Europhys. Lett. 8215001

[9] Lisovskiy V A and Yegorenkov V D 1998 J. Phys. D: Appl. Phys. 313349

[10] Fatechand R 1957 Proc. IEE 104489

[11] Pim J A 1949 Proc. IEE 96117
[12] Lisovskiy V A, Yakovin S D and Yegorenkov V D 2000 J. Phys. D: Appl. Phys. 332722

[13] Schonenburger M 1969 IEEE Trans. Power App. Syst. 88100

[14] Hartmann P, Donkó Z, Bánó G, Szalai L and Rózsa K 2000 Plasma Sources Sci. Technol. 9183

[15] Raizer Y P 1991 Gas Discharge Physics (Berlin: Springer)

[16] Wagenaars E, Bowden M and Kroesen G 2005 Plasma Sources Sci. Technol. 14342

[17] Varela A A 1947 Phys. Rev. 71124

[18] Kirchner F 1947 Phys. Rev. 72348

[19] Kirchner F 1925 Ann. Phys. (Lp3.) 77287

[20] Brown S C 1956 Encyclopedia of Physics vol 22, ed S Flugge (Berlin: Springer) p 558

[21] Bhattacharjee B and Das S P 1974 Indian J. Pure Appl. Phys. 12760

[22] Shoji M and Sato M 1998 Extended Abstracts of the 4th Int Conf. on Reactive Plasmas (Maui, HI) p 81

[23] Shoji M and Sato M 1999 J. Phys. D: Appl. Phys. 321640

[24] Radmilovic-Radjenovic M and Radjenovic B 2006 Plasma Sources Sci. Technol. 151

[25] Lisovsky V A and Yegorenkov V D 1994 J. Phys. D: Appl. Phys. 272340

[26] Lisovskiy V, Kharchenko N and Yegorenkov V 2008 J. Phys. D: Appl. Phys. 41125207

[27] Levitskii S M 1958 Sov. Phys._Tech. Phys. 2887

[28] Hohn F, Jacob W, Beckmann R and Wilhelm R 1997 Phys. Plasmas 4940

[29] Vaughan J R M 1988 IEEE Trans. Electron Devices 351172

[30] Puech V and Torchin L 1986 J. Phys. D: Appl. Phys. 192309

[31] Roznerski W 1996 J. Phys. D: Appl. Phys. 29614

[32] Spriggs K R and Fletcher J 1982 J. Phys. D: Appl. Phys. 151935

[33] Fletcher J and Reid I D 1980 J. Phys. D: Appl. Phys. 132275

[34] Stojanovic V D and Petrovic Z Lj 1998 J. Phys. D: Appl. Phys. 31834

[35] Ferreira C M and Loureiro J 1983 J. Phys. D: Appl. Phys. 16 1611

[36] Nakamura Y and Kurachi M 1988 J. Phys. D: Appl. Phys. 21 718

[37] Lisovskiy V, Booth J P, Landry K, Douai D, Cassagne V and Yegorenkov V 2006 J. Phys. D: Appl. Phys. 391866

[38] Kihara T 1952 Rev. Mod. Phys. 2445 